\title{
ONCOLOGICAL PRINCIPLES IN EMERGENCY SURGERY FOR COLORECTAL CANCER
}

\author{
Sopuev A. ${ }^{1}$, Sydykov N. ${ }^{2}$, Kalzhikeev A. ${ }^{3}$, Samakov A. ${ }^{4}$, Murzakalykov K. ${ }^{5}$ \\ ОНКОЛОГИЧЕСКИЕ ПРИНЦИПЫ В ЭКСТРЕННОЙ ХИРУРГИИ \\ КОЛОРЕКТАЛЬНОГО РАКА \\ Сопуев А. А. ${ }^{1}$, Сыдыков Н. ж. ${ }^{2}$, Калжикеев А. А. ${ }^{3}$, Самаков А. А. ${ }^{4}$, \\ Мурзакалыков К. И. ${ }^{5}$
}

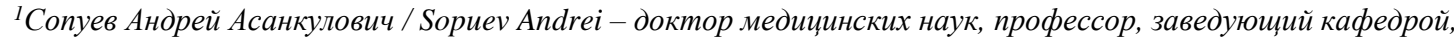 \\ кафедра госпитальной хирургии, \\ Кыргызская государственная медиичнская академия; \\ ${ }^{2}$ Съдыков Нурлан Женишбекович / Sydykov Nurlan - кандидат медицинских наук, заведующий отделением, \\ приемное отделение; \\ ${ }^{3}$ Калжскеев Алмаз Абдрасулович / Kalzhikeеv Almaz-врач-ординатор, \\ Национальный хирургический изентр; \\ ${ }^{4}$ Самаков Алмаз Асанбекович / Samakov Almaz - кандидат медицинских наук, доиент, \\ кафедра госпитальной хирургии, \\ Кыргызская государственная медиичнская академия; \\ ${ }^{5}$ Мурзакальков Кутман Исаевич / Murzakalykov Kutman - врач-ординатор, \\ Национальный хирургический цуентр, г. Биикек, Кыргызская Республика
}

\begin{abstract}
Аннотация: в работе определень возможности соблюдения интраоперационных онкологических принципов у больных с колоректальным раком, осложнившимся кишечной непроходимостью или перфорацией, и прооперированных по экстренным показаниям в клинике неотложной хирургии. В экстренной хирургии рака ободочной кишки выполнимо соблюдение следующих онкологических принципов при резекциях кишечника: (1) эквивалентный объем резекции, (2) микроскопия резекционных срезов и (3) диссекция лимфатических узлов. В экстренной хирургии рака ободочной кишки доля осложнений и летальных исходов была выше, однако, это связано с тяжестью состояния паџиентов и в связи с этим развитием других характерных осложнений, а не с соблюдением онкологических принщипов при хирургических вмешательствах на толстой кишке.

Abstract: the study shows the outcome of an emergency surgery of colorectal cancer was similar to those found in the literature. It was possible to respect the principles of oncologic resection, as regards the extent of resection, surgical margins and lymph node dissection. The morbidity and mortality were higher, however, the different rates were attributed to further complications of the disease and the clinical condition of some of the patients than to the fact those patients had undergone a left colectomy and sigmoidectomy. In the group of patients submitted to right colectomy, we have observed a higher rate of dehiscence of the ileo-transverse anastomosis, superior to the percentage reported in the literature. It has been possible to respect the oncologic principles of resection in the emergency surgery for colorectal cancer.
\end{abstract}

Ключевые слова: колоректальный рак, резекиия толстой кишки.

Keywords: colorectal cancer, colon resection.

Колоректальный рак (КРР) является третьим по распространенности типом злокачественной опухоли и вторым по количеству летальных исходов от рака [2]. Ежегодно в мире фиксируется приблизительно 850 тыс. новых случаев КРР и 500 тыс. летальных исходов [7].

Несмотря на проводимые мероприятия по профилактике и раннему выявлению КРР 6 - 30\% пациентов госпитализируются с клиникой поздних осложнений КРР, которые требовали экстренного хирургического вмешательства $[5,6,8]$.

С учетом этих обстоятельств, один из аспектов, который требует рассмотрения, является проблема соблюдения онкологических принципов резекции кишечника у больных с раком ободочной кишки, подвергшихся экстренному хирургическому вмешательству.

Целью исследования явилось определение возможности соблюдения интраоперационных онкологических принципов у больных с КРР, осложнившемся кишечной непроходимостью или перфорацией, прооперированных по экстренным показаниям в клинике неотложной хирургии. В данном исследовании проводилась оценка объема резекций, микроскопический анализ резецированных краев кишечника и региональных лимфатических узлов.

Также был проведен анализ демографической характеристики пациентов, наблюдаемых осложнений, связанных с хирургическими вмешательствами и долгосрочной выживаемости этих больных. 
В исследование включено 87 пациентов, поступивших в клинику неотложной хирургии, с аденокарциномой толстой кишки или проксимальных отделов прямой кишки. Все эти больные были представлены к хирургической резекции.

Пациенты с первичной опухолью в средней или нижней части прямой кишки, а также больные, у которых, по каким-либо причинам, решено не производить резекцию толстой кишки, в исследование включены не были.

В таблице 1 представлены демографические данные пациентов, включенных в исследование.

Таблииа 1. Демография и факторы риска

\begin{tabular}{|l|c|}
\hline Средний возраст & $60(24-89)$ \\
\hline Системная артериальная гипертензия & $34 / 87$ \\
\hline Сахарный диабет & $6 / 87$ \\
\hline Хроническая обструктивная болезнь легких & $1 / 87$ \\
\hline ASA & \\
\hline I & $39 / 87(45 \%)$ \\
\hline II & $38 / 87(44 \%)$ \\
\hline III & $8 / 87(9 \%)$ \\
\hline IV & $2 / 87(2 \%)$ \\
\hline
\end{tabular}

\section{Результаты.}

В исследовании преобладала локализация рака в сигмовидной кишке и в правой половине толстой кишки (Табл. 2). Кишечная непроходимость наблюдалась у 67 больных (77\%), перфорация кишечника - у 20 больных (23\%). Кровотечения не наблюдались.

Таблица 2. Расположение первичной опухоли

\begin{tabular}{|c|c|c|}
\hline Локализация & $\mathbf{N}$ & $\mathbf{\%}$ \\
\hline Правая половина толстой кишки & 37 & 42,6 \\
\hline Поперечная ободочная кишка & 5 & 5,8 \\
\hline Нисходящая часть толстой кишки & 14 & 16 \\
\hline Сигмовидная кишка & 27 & 31 \\
\hline Верхний отдел прямой кишки & 4 & 4,6 \\
\hline
\end{tabular}

При резекции кишечника первичный анастомоз был наложен у 48 больных (55\%), в остальных 45\% случаев резекции кишечника заканчивали формированием наружной кишечной стомы (Табл. 3). При операциях на правой половине толстой кишки у 89\% пациентов были наложены первичные анастомозы, в 12\% случаев имела место несостоятельность сформированного соустья. При операциях на левой половине толстой кишки первичный анастомоз был наложен в $27 \%$ случаев, эпизодов несостоятельности анастомоза не встречалось. При операциях на сигмовидной кишке и верхней части прямой кишки первичный анастомоз был наложен в 5-ти (16,6\%) случаях без каких-либо осложнений.

Таблица 3. Характеристика проведенных хирургических вмешательств

\begin{tabular}{|c|c|c|c|c|}
\hline \multirow[t]{2}{*}{ Правосторонняя гемиколэктомия } & Анастомозирование & 33 & $\begin{array}{c}\text { Несостоятельность } \\
\text { анастомоза }\end{array}$ & $\begin{array}{c}4 \\
(12 \%)\end{array}$ \\
\hline & Колостомия & 4 & & \\
\hline \multirow[t]{2}{*}{ Левосторонняя гемиколэктомия } & Анастомозирование & 3 & $\begin{array}{c}\text { Несостоятельность } \\
\text { анастомоза }\end{array}$ & 0 \\
\hline & Колостомия & 8 & & \\
\hline \multirow[t]{2}{*}{ Резекция сигмовидной кишки } & Анастомозирование & 5 & $\begin{array}{c}\text { Несостоятельность } \\
\text { анастомоза }\end{array}$ & 0 \\
\hline & Колостомия & 25 & & \\
\hline \multirow{2}{*}{$\begin{array}{c}\text { Резекция поперечно-ободочной } \\
\text { кишки }\end{array}$} & Анастомозирование & 2 & $\begin{array}{c}\text { Несостоятельность } \\
\text { анастомоза }\end{array}$ & $\begin{array}{c}1 \\
(50 \%)\end{array}$ \\
\hline & Колостомия & 2 & & \\
\hline
\end{tabular}




\begin{tabular}{|l|l|l|c|c|}
\hline Тотальная колэктомия & Анастомозирование & 5 & $\begin{array}{c}\text { Несостоятельность } \\
\text { анастомоза }\end{array}$ & 0 \\
\hline
\end{tabular}

В 7-ми (8\%) случаях при гистологическом исследовании срезов операционного материала обнаружены скомпрометированные участки. У этих пациентов обнаружены объемные Т4 опухоли: у 4-х пациентов опухоли локализовались в верхнем отделе прямой кишки, в 3-х случаях - в правой половине толстой кишки. У 4-х из этих пациентов имела место перфорация кишечника, представленная в качестве основного симптома заболевания. У этой категории больных исключались мультивисцеральные резекции единым блоком из-за плохого клинического состояния больных.

Гистологический анализ степени клеточной дифференцировки резецированного материала показал хорошо дифференцированные опухоли у 63 больных (72\%), умеренно дифференцированные аденокарциномы у 15 (17\%) больных и слабо дифференцированные аденокарциномы у 9 пациентов $(11 \%)$.

У 71\% пациентов, перенесших удаление опухоли, количество иссеченных региональных лимфатических узлов было равно или больше 12-ти со средним общим значением 4,02 метастатических лимфатических узла.

В группе оперированных больных несостоятельность анастомоза имела место в 10,4\% случаев, эвентрация у 2,2\% пациентов, септический шок у 5,7\% больных и нагноение послеоперационных ран у 6,8\% больных (Табл. 4). Показатели общей выживаемости приведены в таблицах $\underline{5}, \underline{6}$.

Таблица 4. Характеристика осложнений

\begin{tabular}{|c|c|c|}
\hline Осложнения & $\mathbf{N}$ & $\mathbf{\%}$ \\
\hline Несостоятельность анастомоза & 5 & 10,4 \\
\hline Эвентрация & 2 & 2,2 \\
\hline Инфицирование послеоперационных ран & 6 & 6,8 \\
\hline Септический шок & 5 & 5,7 \\
\hline Пневмония & 3 & 5,7 \\
\hline Колостомические осложнения & 2 & 5,1 \\
\hline
\end{tabular}

Таблица 5. Распределение больных, оперированных по экстренным показаниям, по классификации TNM (AJCC / UІСС 6-е издание)

\begin{tabular}{|c|c|c|}
\hline Стадия & $\mathbf{N}$ & \% \\
\hline I & 1 & 1,1 \\
\hline II a & 11 & 12,7 \\
\hline II b & 4 & 4,6 \\
\hline III a & 4 & 4,6 \\
\hline III b & 17 & 19,6 \\
\hline III c & 12 & 13,8 \\
\hline IV & 38 & 43,6 \\
\hline
\end{tabular}

Таблица 6. Общзая трех- и пятилетняя выживаемость в соответствии с АJCC / UICC, 6-е изд.

\begin{tabular}{|c|c|c|}
\hline Стадия & Трехлетняя общая выживаемость & Пятилетняя общая выживаемость \\
\hline I & $100,0 \%$ & $74 \%$ \\
\hline II & $50,0 \%$ & $32 \%$ \\
\hline III & $33 \%$ & $25 \%$ \\
\hline IV & $10 \%$ & $5 \%$ \\
\hline
\end{tabular}

Средние сроки пребывания в отделении интенсивной терапии составили 5, 7 суток, послеоперационные осложнения и периоперационная смертность (в течение 30 дней после операции) были равны $33,6 \%$ и $20 \%$, соответственно.

Обсуждение.

В проведенном исследовании у 77\% пациентов рак ободочной кишки осложнился кишечной непроходимостью, у остальных больных заболевание осложнилось перфорацией толстой кишки. Почти у половины (42,6\%) пациентов опухоль локализовалась в правой половине толстой кишки, у $36 \%$ пациентов опухоль локализовалась в сигмовидной кишке и в верхней части прямой кишки.

Как правило, экстренное хирургическое вмешательство приводило к более высоким показателям количества осложнений, смертности и плохого прогноза в сравнении с плановыми хирургическими вмешательствами. Общая выживаемость и процент выживаемости был ниже у больных с КРР, которые подверглись экстренным хирургическим вмешательствам. 
В нашем исследовании из 87-ми пациентов, мы также наблюдали более высокие показатели заболеваемости и смертности: $33,6 \%$ и $20 \%$ соответственно.

При правосторонней колэктомии несостоятельность анастомоза встречалась в $12 \%$ случаев, что выше, чем в исследованиях других авторов [9].

Другим важным аспектом лечения КРР в условиях неотложной хирургии является возможность соблюдения следующих онкологических принципов при резекциях кишечника: (1) объем резекции и канцеронегативные хирургические срезы, (2) резекция единым блоком опухоли и прилежащих к ней тканей, (3) лимфодиссекция, по меньшей мере, 12-ти региональных лимфатических узлов, осмотренные морфологом [1, 4]. Остается неясным, могут ли на самом деле онкологические принципы хирургии быть реализованы в алгоритме экстренных операций? В настоящее время в некоторых научных исследованиях демонстрируются усилия по соблюдению этих принципов у пациентов с осложненной формой рака толстой кишки $[1,4,10,11]$. Высказывается мнение о том, что принципы онкологической резекции при экстренной операции по поводу КРР могут быть соблюдены, а также могут быть достигнуты приемлемые результаты по долгосрочной выживаемости этих больных [3]. При решении вопроса о выполнении неотложной операции с соблюдением онкологических принципов необходимо учитывать некоторые клинические и хирургические факторы, негативно влияющие на выбор онкологической резекции. Это риск усугубления тяжести состояния пациента; возможное расширение объема и времени хирургического вмешательства; сложность выполнения адекватной лимфодиссекции; технические сложности мобилизации и манипулирования с растянутой толстой кишкой; выраженный перитонит в случаях, связанных с перфорацией кишечника.

Было высказано предположение, что при КРР хирургическое вмешательство, выполненное узким специалистом, может оказать существенное влияние на выживаемость [1]. Хирурги, оказывающие неотложную помощь, имеют различную степень специализации. Тем не менее, большинство из них обладают меньшей специальной подготовкой и могут выполнить резекцию толстой кишки только для решения неотложной ситуации без соблюдения онкологических принципов.

Первый критерий, который рассматривался в нашем исследовании, это объем резекции и патологическое состояние краев резекции. В общем, этот критерии может быть соблюден даже в экстренной ситуации: (1) резецируемые отступы от краев патологического процесса на расстоянии 5 - 10 см, (2) удаление сальниковых и параколических лимфатических узлов, а также (3) сведение к минимуму риска развития несостоятельности анастомоза. В исследуемой группе больных $92 \%$ пациентов после резекции патологического очага имели классификационную категорию R0 (состояние опухоли после лечения). В 8\% случаев были обнаружены положительные микроскопические поля по резекционному срезу, что было связано с опухолями Т4, невозможностью мультивисцеральной резекции единым блоком и тяжелым клиническим состоянием больных. Вторым критерием, который рассматривался в исследовании, явилась диссекция лимфатических узлов, которая имеет прогностическое и терапевтическое значение. Удаление всех метастатических лимфатических узлов также имеет классификационную категорию R0 и как стандарт, по крайней мере, удаление 12-ти лимфатических узлов, необходимых для обеспечения соответствующей точности. В исследуемой группе $71 \%$ пациентов соответствовали этому критерию. Абсолютное число диссекцированных лимфатических узлов зависит от объема резекции и было выше у пациентов, перенесших тотальную или субтотальную колэктомию.

\section{Выводы}

В экстренной хирургии рака ободочной кишки выполнимо соблюдение следующих онкологических принципов при резекциях кишечника: (1) эквивалентный объем резекции, (2) микроскопия резекционных срезов и (3) диссекция лимфатических узлов.

В экстренной хирургии рака ободочной кишки доля осложнений и летальных исходов была выше, однако, это связано с тяжестью состояния пациентов и в связи с этим развитием других характерных осложнений, а не с соблюдением онкологических принципов при хирургических вмешательствах на толстой кишке.

\section{Лumepamypa}

1. Chang G. J., Rodriguez-Bigas M. A., Skibber J. M., Moyer V. A: Lymph node evaluation and survival after curative resection of colon cancer: systematic review. J. Natl. Canc. Inst., 2007. 99(6):433-41.

2. Jemal A., Siegel R., Ward E., Murray T., Xu J., Thun M. J.: Cancer statistics, 2007. CA Cancer J. Clin., 2007. 57: 43-66.

3. MacArdle C.S., Hole D. J.: Influence of volume and specialization on survival following surgery for colorectal cancer. Br. J. Surg, 2004. 91: 610-7.

4. Nelson H., Petrelli N., Carlin A., Couture J., Fleshman J., Guilhem J.: Guidelines 2000 for Colon and Rectal Cancer Surgery. J. Natl. Canc. Inst., 2001. 93. 
5. Phang P. T., MacFarlane J. K., Taylor R. H., Chiefetz R., Davis N., Hay J.: Effect of emergent presentation on outcome from rectal cancer management. Am J. Surg., 2003. 185: 450-4.

6. Ponz de Leon M., Sassatelli R., Scalmati A., Di Gregorio C., Fante R., Zanguieri G.: Descriptive epidemiology of colorectal câncer in Italy: the 6 year experience of a specialized registry. Eur J. Cancer, 1993. 29(3):367-71.

7. Ries LAG, Eisner M. P.: SEER Cancer Statistics Review, 1973-1997. Bethesda M. D.: National Cancer Institute; 2000. Google Scholar.

8. Scott N. A., Jeacock J., Kingston R. D.: Risk factors in patients presenting as an emergency with colorectal cancer. Br. J. Surg., 1995. 82: 321-3.

9. Wyrzycovski A., Feliciano D. V., George T. A., Tremblay L. N., Rozycki G. S., Murphy T. W. et al.: Emergent right hemicolectomies. Am Surg., 2005. 71 (8):653-6.

10. Сопуев А.А., Абдиев А.Ш., Калжикеев А. А., Сыдыков Н.Ж., Мамбетов А. К. Профилактическое дренирование брюшной полости после операций на дистальных отделах ЖКТ // Проблемы современной науки и образования, 2016. № 13 (55). С. 129-131.

11. Сопуев А. А., Исаев Д. К., Сыдыгалиев К. С., Сыдыков Н. Ж., Мамбетов А. К. Значение анатомических особенностей сигмовидной кишки в развитии ее заворота // Проблемы современной науки и образования, 2016. № 21 (63). С. 97-99. 